\title{
Newly translated proteins are substrates for ubiquitin, ISG15, and FAT10
}

\author{
Valentina Spinnenhirn ${ }^{1}$, Annegret Bitzer ${ }^{1}$, Annette Aichem ${ }^{2}$ and Marcus Groettrup ${ }^{1,2}$ \\ 1 Division of Immunology, Department of Biology, University of Konstanz, Germany \\ 2 Biotechnology Institute Thurgau at the University of Konstanz, Kreuzlingen, Switzerland
}

\begin{abstract}
The ubiquitin-like modifier, FAT10, is involved in proteasomal degradation and antigen processing. As ubiquitin and the ubiquitin-like modifier, ISG15, cotranslationally modify proteins, we investigated whether FAT10 could also be conjugated to newly synthesized proteins. Indeed, we found that nascent proteins are modified with FAT10, but not with the same preference for newly synthesized proteins as observed for ISG15. Our data show that puromycinlabeled polypeptides are strongly modified by ISG15 and less intensely by ubiquitin and FAT10. Nevertheless, conjugates of all three modifiers copurify with ribosomes. Taken together, we show that unlike ISG15, ubiquitin and FAT10 are conjugated to a similar degree to newly translated and preexisting proteins.
\end{abstract}

Keywords: cotranslational conjugation; defective ribosomal products; FAT10; ISG15; newly translated proteins; ubiquitin

Quality control pathways that maintain homeostasis of the cellular proteome deal with long lived as well as rapidly degraded proteins (RDPs). The group of RDPs is either short-lived proteins that have achieved a stable conformation but for functional reasons are tightly regulated by the ubiquitin proteasome system (UPS) or they are degraded because they fail to incorporate into multisubunit complexes [1]. RDPs also comprise aggregation-prone defective ribosomal products (DRiPs). These are proteins that are unable to achieve a stable conformation due to folding problems or translation errors. Moreover, RDPs include unfinished translation products that are liberated from stalled ribosomal complexes via cotranslational quality control mechanisms [2]. Previous studies investigating the fate of newly translated RDPs found that a percentage of up to $30 \%$ of all nascent chains are degraded by the proteasome within half an hour after their synthesis [3]. Although this number was subsequently challenged by other investigators [4], there is broad agreement on the fact that the UPS degrades a substantial part of newly synthesized proteins. Whether this is primarily a consequence of the actual failure rate during expression and folding of proteins remains controversial [5].

The complexity of this quality control pathway increased when other investigators defined distinct subpopulations of cotranslationally ubiquitylated and rapidly degraded substrates [6]. In addition, the ubiquitin-like modifier (ULM), ISG15, was reported to be cotranslationally conjugated to nascent chains in analogy to ubiquitin [7]. In this context, substrate independent, cotranslational ISGylation was proposed to fulfill an antiviral function in line with the fact that the expression of ISG15 as well as its conjugation enzyme cascade is strongly induced by IFN- $\alpha$ and

\section{Abbreviations}

ALIS, aggresome-like induced structures; DC, dendritic cell; DRiPs, defective ribosomal products; FAT10, HLA-F adjacent transcript 10; HLA, human leukocyte antigen; ISG15, interferon-stimulated gene 15; Ltn, listerin; RDPs, rapidly degraded proteins; ULM, ubiquitin-like modifier; UPS, ubiquitin proteasome system. 
IFN- $\beta$. The antiviral effect was reported to be mediated by interfering with oligomerization of viral proteins leading to a dominant-inhibitory effect on virulence. However, no evidence was reported that would indicate an influence of ISG15 on the degradation of virus-derived proteins or antigenic processing.

Interestingly, the ULM FAT10 which structurally resembles ISG15 targets substrates for proteasomal degradation in a ubiquitin-independent manner [8]. FAT10 is inducible by the proinflammatory cytokines IFN- $\gamma$ and TNF- $\alpha$ and has been implicated in antigen processing as it is encoded in the MHC locus, is expressed constitutively in lymphoid tissues, and is induced during dendritic cell (DC) maturation [9]. Moreover, it was shown that fusion to FAT10 enhanced the MHC class I-restricted presentation of viral proteins $[10,11]$.

These observations prompted us to assess whether FAT10 may function as a cotranslational modifier possibly linking protein synthesis and antigen processing. We therefore investigated FAT10 side by side with the known cotranslational modifiers, ubiquitin and ISG15, using several previously applied technical approaches [6,7]. From our results, we conclude that in contrast to ISG15, which preferentially modifies newly translated proteins, FAT10 and ubiquitin are conjugated equally well to newly and formerly translated proteins.

\section{Materials and methods}

\section{Cells, plasmids, and antibodies}

Transfection of HEK293T and HEK293 cells was performed with TransIT ${ }^{\circledR}-$ LT1 Transfection Reagent (Mirus, Madison, WI, USA) according to the manufacturer's instruction with a $1: 3$ ratio of DNA to reagent. For FAT10 expression, pcDNA3-His-3xFlag-FAT10-wt (FAT10-GG) [12] and pcDNA3-His-3xFlag-FAT10 $\Delta$ GG (FAT10-AV) [13] were used. For cysteine-less FAT10 expression, pcDNA3-His3xFlag-FAT10-GG/AV were modified by mutating all cysteines to serines (FAT10-C0-GG/AV). Tagged ubiquitin was expressed by pcDNA3.1-HA-ubiquitinK48R $\Delta$ GG (Ub-GA) and pcDNA3.1-HA-ubiquitin (Ub-GG) [8]. Other plasmids were previously described: pcDNA-Ube1L [14], pcDNAUbcH8 [14], pc3xFlag-ISG15-C0 [7], pTriEx-2-S-hHerc5 [15], and p3xFlag-ubiquitin [16].

Tagged proteins were detected with HRP-conjugated anti-Flag (M2; Sigma, Darmstadt, Germany) or anti-HA (HA7; Sigma) monoclonal antibodies. Monoclonal mouse antibodies were also used for GAPDH (71.1; Sigma) and human FAT10 (4F1) [13]. Rabbit polyclonal antibodies were used for detection of Herc5 (BML-PW0920; Enzo Life Sciences, Lörrach, Germany), Ube1 (BML-PW8390; Enzo),
Rpl7 (ab72550; Abcam, Cambridge, UK), Use1 [13], puromycin (kind gift from Peter Walter, UCSF), and Uba6 (PW0525; Biomol, Hamburg, Germany).

\section{Radiolabeling and puromycin labeling}

HEK293T or HEK293 cells were radiolabeled with ${ }^{35} \mathrm{~S}-\mathrm{L}$ cysteine. Cells were washed with PBS and incubated prior to labeling for $1 \mathrm{~h}$ with starvation medium (RPMI lacking L-glutamine, cysteine, and methionine, 10\% dialyzed FBS, $1 \%$ penicillin and streptomycin, $300 \mu \mathrm{g} \cdot \mathrm{mL}^{-1}$ L-glutamine). This starvation medium was replaced by labeling medium (starvation medium supplemented with $15 \mu \mathrm{g} \cdot \mathrm{mL}^{-1} \mathrm{~L}$ methionine and $0.25 \mu \mathrm{Ci}$ Cys- $\left[{ }^{35} \mathrm{~S}\right]$ ) for $2 \mathrm{~h}$. The labeling medium was either replaced by full medium (E) or cells were harvested (L). Samples were processed for SDS/PAGE and gels were subsequently dried, and exposed to imaging plates (Fuji Film).

For puromycin experiments, transiently transfected HEK293T or HEK293 cells were treated $24 \mathrm{~h}$ post transfection with puromycin (10 $\mu \mathrm{M}$ final concentration) for 10 min. Total cell extracts were prepared with Ripa buffer [50 mm Tris, pH 7.5, $1 \mathrm{~mm}$ EDTA, $150 \mathrm{~mm} \mathrm{NaCl}$, $0.1 \%$ SDS, $1 \%$ NP-40, $5 \mu \mathrm{M}$ MG-132, $1 \times$ protease inhibitor cocktail (complete Mini EDTA-free; Roche, Mannheim,Germany)] for $30 \mathrm{~min}$ on ice and lysates were cleared by centrifugation at $20000 \mathrm{~g}$ for $15 \mathrm{~min}$ at $4{ }^{\circ} \mathrm{C}$. Lysates were subsequently processed for immunoprecipitation experiments. For experiments in Fig. 2C, threefold more cells were used than in experiments shown in Fig. 2A,B.

\section{Immunoprecipitation and immunoblot}

After two cycles of washing with PBS, cells were lysed in NP-40 lysis buffer [20 mm Tris pH $7.8,50 \mathrm{~mm} \mathrm{NaCl}$, $10 \mathrm{~mm} \mathrm{MgCl} 2,1 \% \mathrm{NP}-40,1 \times$ protease inhibitor cocktail (complete Mini EDTA-free; Roche)] for $30 \mathrm{~min}$ on ice after rigorous vortexing. The lysate was cleared at $20000 \mathrm{~g}$ for $15 \mathrm{~min}$ at $4{ }^{\circ} \mathrm{C}$. For radioactive experiments, lysates were adjusted to the same relative radioactivity by scintillation counting (TOPcount NXT; Canberra Packard, Schwadorf, Austria) and incubated with $30 \mu \mathrm{L}$ pre-equilibrated EzViewTM Red anti-Flag affinity gel (Sigma-Aldrich) at $4{ }^{\circ} \mathrm{C}$ for $3 \mathrm{~h}$. For Flag-FAT10 conjugate immunoprecipitation, Flag beads were washed twice with NP-40 lysis buffer and subsequently incubated with $500 \mu \mathrm{L}$ of NP-40 lysis buffer containing $100 \mu \mathrm{g} \cdot \mathrm{mL}^{-1} 3 \times$ Flag peptide (SigmaAldrich) for $45 \mathrm{~min}$ at $30^{\circ} \mathrm{C}$ and spun down. This step was repeated twice. Eluates were pooled and subsequently incubated with $30 \mu \mathrm{L}$ pre-equilibrated EzViewTM Red Protein $G$ affinity gel (Sigma-Aldrich) with $8 \mu \mathrm{g}$ human FAT10-specific antibody $4 \mathrm{~F} 1$ overnight at $4{ }^{\circ} \mathrm{C}$. Beads were washed twice with $1 \mathrm{~mL}$ NET-TN (50 mm Tris $\mathrm{pH} 8$, $650 \mathrm{~mm} \mathrm{NaCl}, 5$ mм EDTA, 0.5\% Triton X-100) and twice 
with $1 \mathrm{~mL}$ NET-T (50 mm Tris $\mathrm{pH} 8,150 \mathrm{~mm} \mathrm{NaCl}, 5 \mathrm{~mm}$ EDTA, $0.5 \%$ Triton X-100). Beads were boiled for $5 \mathrm{~min}$ at $95{ }^{\circ} \mathrm{C}$ in $2 \mathrm{xSDS}$ sample buffer with a final concentration of $10 \%(\mathrm{v} / \mathrm{v}) \beta$-mercaptoethanol. Samples were separated by SDS/PAGE and transferred onto a nitrocellulose membrane.

Lysates prepared from puromycin-treated cells were incubated with $20-30 \mu \mathrm{L}$ pre-equilibrated EzViewTM Red anti-Flag affinity gel (Sigma-Aldrich) at room temperature for $2 \mathrm{~h}$. The Flag beads were washed six times with Ripa buffer and subsequently boiled for $5 \mathrm{~min}$ at $95{ }^{\circ} \mathrm{C}$ in $4 \times$ SDS sample buffer with a final concentration of $10 \%$

(v/v) $\beta$-mercaptoethanol. Samples were separated by SDS/ PAGE and transferred onto a nitrocellulose membrane.

Autoradiographic and immunoblot signals were quantified by IMAges (Wayne Rasband, National Institutes of Health, Bethesda, MD, USA). Autoradiographic signals of immunoprecipitations were normalized to autoradiographic load signals. The autoradiographic signal of untransfected controls was subtracted and these values were subsequently normalized to immunoblot signals of respective immunoprecipitations.

\section{Ribosome purification}

All solutions were sterile filtered and kept at $4{ }^{\circ} \mathrm{C}$. Confluent HEK293T cells from a $15 \mathrm{~cm}$ dish were harvested and lysed in $400 \mu \mathrm{L}$ lysis buffer $[250 \mathrm{~mm}$ sucrose; $25 \mathrm{~mm} \mathrm{KCl} ; 5 \mathrm{~mm}$ $\mathrm{MgCl}_{2} ; 50 \mathrm{~mm}$ Tris pH 7.4, 0.7\% NP-40, Protease inhibitors (Roche complete EDTA-free), Phosphatase inhibitor (Roche PhosStop), $2 \mathrm{~mm}$ dithioerythritol (DTE)]. Lysates were centrifuged at $800 \mathrm{~g}$ at $4{ }^{\circ} \mathrm{C}$ for $10 \mathrm{~min}$. The supernatant (postnuclear fraction) was subsequently centrifuged at $12400 \mathrm{~g}$ at $4{ }^{\circ} \mathrm{C}$ for $10 \mathrm{~min}$. Input samples were taken and the postmitochondrial supernatant was layered on $1 \mathrm{~mL}$ of sucrose cushion ( $1 \mathrm{~m}$ sucrose, $25 \mathrm{~mm} \mathrm{KCl}, 5 \mathrm{~mm}$ $\mathrm{MgCl}_{2}, 50 \mathrm{~mm}$ Tris $\mathrm{pH}$ 7.4) filled into polycarbonate centrifuge tubes $(1.5 \mathrm{~mL}$ capacity; Beckmann, Krefeld, Germany) and centrifuged at $305000 \mathrm{~g}$ for $2 \mathrm{~h}$. The pellet was washed with cold water to remove remaining sucrose and resuspended in $150 \mu \mathrm{L}$ resuspension buffer $[25 \mathrm{~mm} \mathrm{KCl}$, $5 \mathrm{~mm} \mathrm{MgCl} 2,50 \mathrm{~mm}$ Tris $\mathrm{pH} \mathrm{7.4,} \mathrm{Protease} \mathrm{inhibitors}$ (Roche complete EDTA-free), Phosphatase inhibitor (Roche PhosStop), 2 mм DTE].

\section{Results}

\section{ISG15, but neither ubiquitin nor FAT10, are preferentially conjugated to newly translated proteins}

To address the question whether the ULM FAT10 is covalently conjugated to newly translated proteins, we performed radioactive labeling experiments according to a protocol previously applied by Huibregtse and coworkers [7] (for illustration see the experimental scheme depicted in Fig. 1A). They transiently expressed a cysteine-less mutant of Flag-tagged ISG15 (ISG15-C0) as well as the three enzymes necessary for conjugation Ube1L, UbcH8, and Herc5 in HEK293T cells. One fraction of cells was radioactively labeled with ${ }^{35} \mathrm{~S}$-Cys $8 \mathrm{~h}$ after transfection (designated ' $E$ ' for 'early labeling'). Labeled proteins from these E-samples represent mostly fully translated and folded proteins, previously designated 'retirees' [1]. A second sample was labeled $22 \mathrm{~h}$ after transfection (designated ' $L$ ' for 'late labeling') right before harvest, and therefore it mainly contained labeled proteins that were newly translated. Nevertheless, we cannot exclude that proteins labeled at the beginning of the $2 \mathrm{~h}$ labeling period were already degraded at the end of this period. ISG15-C0 itself was unlabeled in any sample due to the Cys to Ser mutation. The authors detected higher autoradiographic signals in L- compared to E-samples indicating that the majority of ISGylated substrates are newly translated proteins [7]. We performed the same experiment (Fig. 1B) and included as a control ISG15-C0 without expression of conjugating enzymes to be able to discriminate between covalent conjugation substrates and noncovalent binding to ISG15-C0 (Fig. 1B, lane 5). Comparable amounts of ISG15-C0 conjugates in E- and L-samples and the lack of ISG15-C0 conjugates in samples where ISG15-C0 was transfected singly were monitored by immunoblot (Fig. 1B lower panel). In conclusion, we could confirm the finding by Durfee et al. [7] of preferential covalent conjugation of ISG15-C0 to newly translated substrates (lane 2 and 4) in three independent experiments.

Fig. 1. ISG15, but not ubiquitin and FAT10, is preferentially conjugated to newly translated proteins. (A) Experimental scheme of early and late metabolic labeling of cells with ${ }^{35}$ S-cysteine subsequent to transient transfection at time point 0 h. (B) HEK293T cells were transiently transfected with Flag-ISG15-GG with and without the corresponding E1, E2, E3 enzymes, Ube1L, UbcH8, and Herc5, or in (C) with HAubiquitin-GG or HA-ubiquitin-GA or in (D) with HIS-Flag-FAT10-C0-GG or HIS-Flag-FAT10-C0-AV. Cells were labeled for 2 h with ${ }^{35}$ S-cysteine either $8 \mathrm{~h}$ after transfection and then chased for $14 \mathrm{~h}$ before lysis (E-samples) or $22 \mathrm{~h}$ after transfection and lysed immediately thereafter (L-samples). Load samples were taken and an anti-Flag or anti-HA immunoprecipitation was performed. For the HIS-Flag-FAT10-C0 immunoprecipitates, an elution with Flag peptide and reprecipitation with the FAT10-specific mAb 4F1 were performed. Samples were subsequently analyzed by autoradiography (upper panels) and immunoblot (lower panels). 
A Flag-ISG15-CO/E1/E2/E3

HIS-Flag-FAT10 CO(GG/AV)

HA-Ubiquitin (GG/GA)

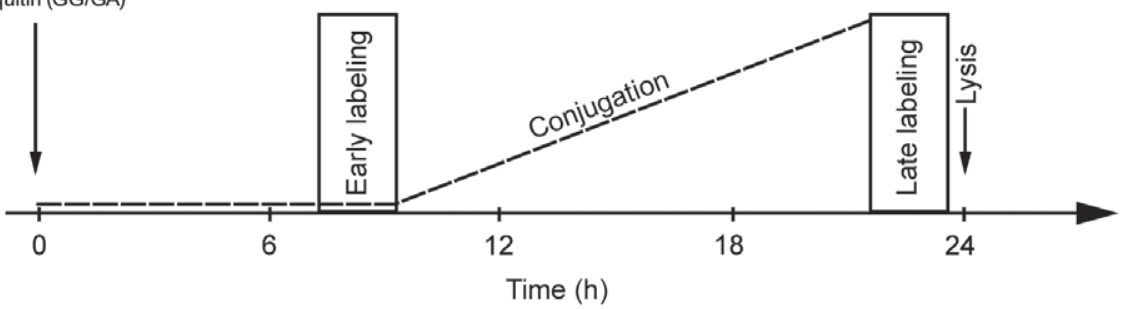

B

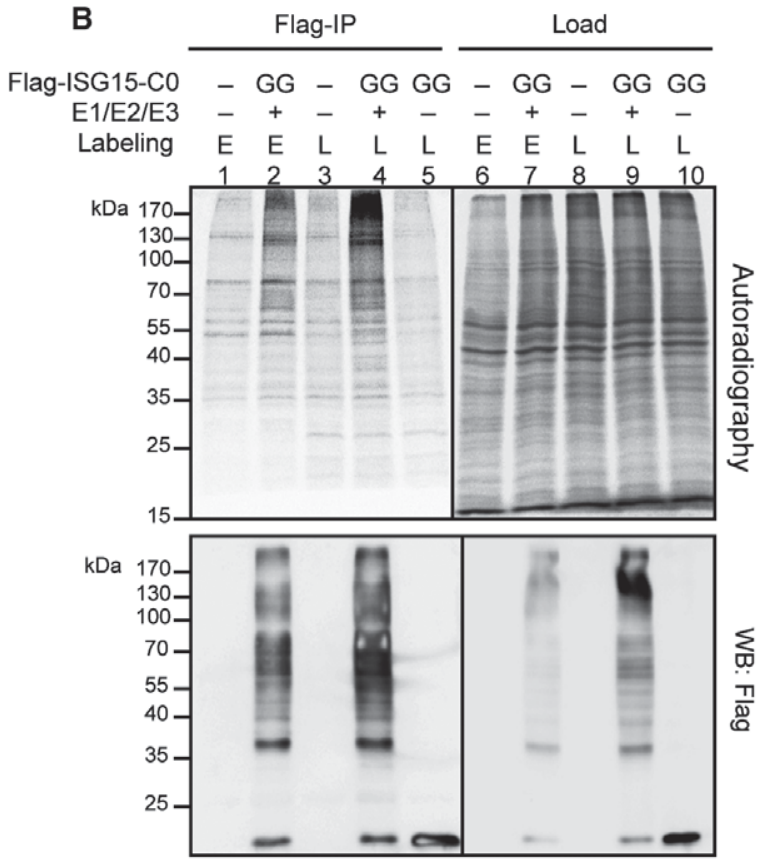

D

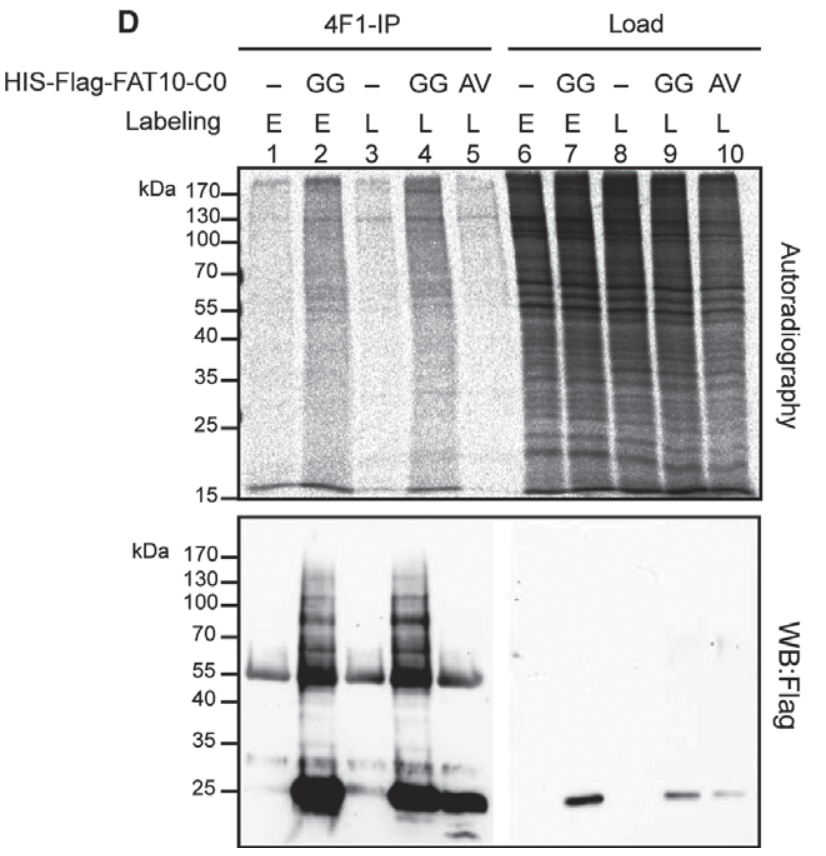

HIS-Flag-FAT10-C0 - GG - GG AV - GG - GG AV

$\begin{array}{lllllllllll}\text { Labeling } & \mathrm{E} & \mathrm{E} & \mathrm{L} & \mathrm{L} & \mathrm{L} & \mathrm{E} & \mathrm{E} & \mathrm{L} & \mathrm{L} & \mathrm{L}\end{array}$

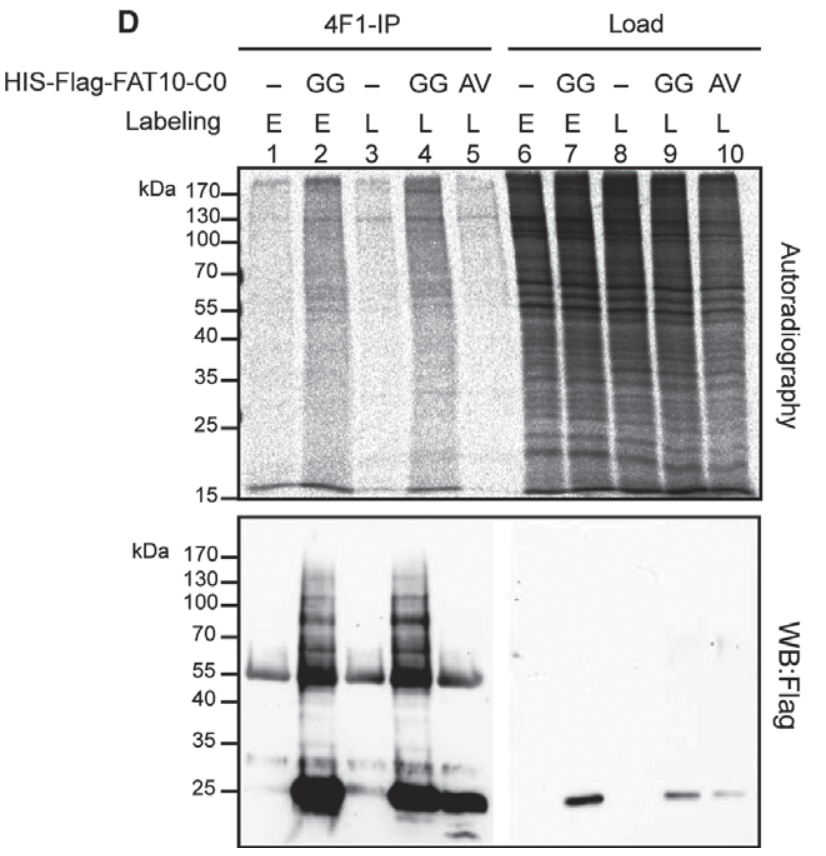

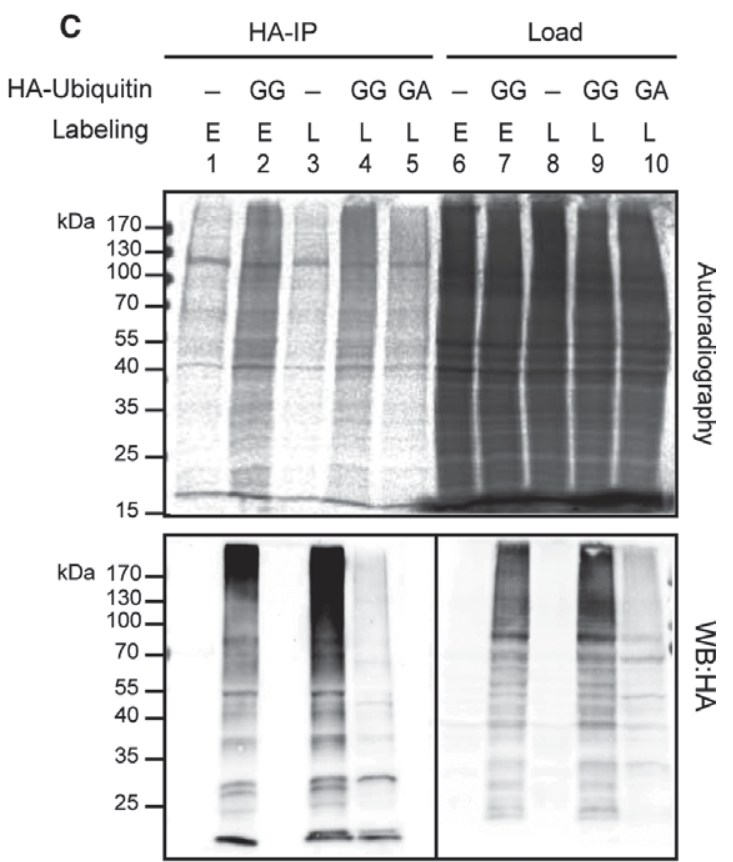


Next, we performed similar experiments for ubiquitin (Ub) and the UBL FAT10 in parallel. To this aim, we transfected cells with Ub-GG as well as Ub-GA, a mutant that cannot be activated and conjugated to substrates (Fig. 1C). Importantly, in the case of ubiquitin, no cysteine to serine mutation was necessary as wild-type ubiquitin lacks cysteine. In contrast to ISG15-C0, no preferential targeting of newly translated proteins by ubiquitin conjugation was detectable. This was evident by similar intensities of autoradiographic signals in E- as well as Llabeled Ub-GG-transfected and -immunoprecipitated samples (Fig. 1C, lane 2 and 4) and only background signals for untransfected and Ub-GA-transfected samples (lane 1, 3, 5). Control immunoblots showed comparable levels of ubiquitin conjugation in both E- and L-labeled Ub-GG samples (Fig. 1C bottom, lane 2 and 4) but only marginal amounts of high molecular weight proteins for the unconjugatable Ub-GA (lane 5). We conclude that although there was no preference of ubiquitin conjugation toward newly translated proteins observable, a substantial fraction of the ubiquitylated proteome indeed represents newly translated proteins.

The same radioactive pulse labeling experiments were also conducted for FAT10. This required the generation of expression constructs encoding conjugatable and nonconjugatable human FAT10 variants in which all four cysteines were replaced by serines. These cysteine-less Flag-tagged FAT10 mutants, FAT10-C0-GG, as well as the nonconjugatable FAT10 mutant, FAT10-C0-AV, were included to be able to discriminate between covalent substrates and noncovalent binding to FAT10. FAT10-C0-GG overexpression, however, generally results in a much higher ratio of FAT10 monomer to conjugates as compared to ubiquitin or ISG15. To optimize the signal-to-noise ratio, we extended the experimental procedure by a second immunoprecipitation to gain a higher yield and purity of FAT10 conjugates. For this aim, immunoprecipitates were eluted from antiFlag beads with 3xFlag peptide and a second immunoprecipitation against human FAT10 with
$\mathrm{mAb} 4 \mathrm{~F} 1$ was performed. With this procedure, labeled FAT10-C0-GG conjugates could be successfully and reproducibly visualized by autoradiography. These experiments showed-in contrast to ISG15 (Fig. 1B) - equal intensities in E- and L-samples for FAT10-conjugated proteins (Fig. 1D, lane 2 and 4). These signals represented covalent conjugates, as the FAT10-C0-AV sample showed no signal over background (lane 5). Immunoblot analysis confirmed that E- and L-samples contained comparable amounts of FAT10-C0-GG conjugates (Fig. 1D lower panel) and no conjugates were detectable, as expected, in untransfected and FAT10-C0-AV-transfected samples (lanes 1, 3, and 5). We conclude that-similar to ubiquitin (Fig. 1C) - also the FAT10 conjugation machinery does target newly translated proteins, but not to a greater extent than 'retirees'.

\section{Puromycin-labeled nascent chains are modified with ISG15}

In a next step, we wanted to validate the results obtained with radioactive labeling in Fig. 1 with a different experimental approach. Therefore we used puromycin, a tRNA analog that inhibits translation by covalent incorporation at the carboxy-terminal end of nascent polypeptide chains leading to chain truncation. If nascent chains are preferential targets of ISG15, ubiquitin or FAT10, those puromycin-labeled nascent chains should be detectable within the targeted proteome of the respective modifier. Therefore, HEK293T cells were transfected either with Flag-tagged ubiquitin, or with Flag-ISG15 in combination with its cognate E1, E2, and E3 enzymes, or with Flag-FAT10, and labeled with a high dose of puromycin for $10 \mathrm{~min}$ in culture (Fig. 2). After Flag immunoprecipitation, samples were subsequently analyzed by anti-puromycin immunoblot. Similar to Durfee et al. [7], we detected a robust puromycin-specific signal for ISG15 conjugates (Fig. 2A/B lanes 3 and 4) and also ubiquitin conjugation to puromycin-labeled polypeptides was apparent (Fig. 2A/B lanes 1 and 2). In contrast, less or no

Fig. 2. Conjugation to nascent chains labeled with puromycin by ISG15, ubiquitin, and FAT10. HEK293T cells were transiently transfected either with Flag-ISG15-GG and its corresponding E1, E2, E3 enzymes, Ube1L, UbcH8 and S-Herc5, or with Flag-ubiquitin-GG or with HISFlag-FAT10-GG as indicated above the panels. One day after transfection, cells were labeled with puromycin for 10 min and subsequently lysed. Then, an anti-Flag immunoprecipitation was performed. Samples were subsequently analyzed by immunoblot to detect (A) puromycin or $(B)$ the Flag tag of the respective modifiers and their conjugates. In figure (C), three times more cells per immunoprecipitation were used as compared to experiments in (A) and (B) and puromycin and Flag-specific western blots of total lysates are shown as well. In addition, in the lanes indicated, the proteasome inhibitor MG132 was added to the cells $1 \mathrm{~h}$ before the start of the 10-min puromycin-labeling period and subsequent harvest of the cells. $\beta$-Actin served as loading control. The asterisks at the upper left panel denote the heavy and light chains of the Flag-specific antibody used for immunoprecipitation. 
A

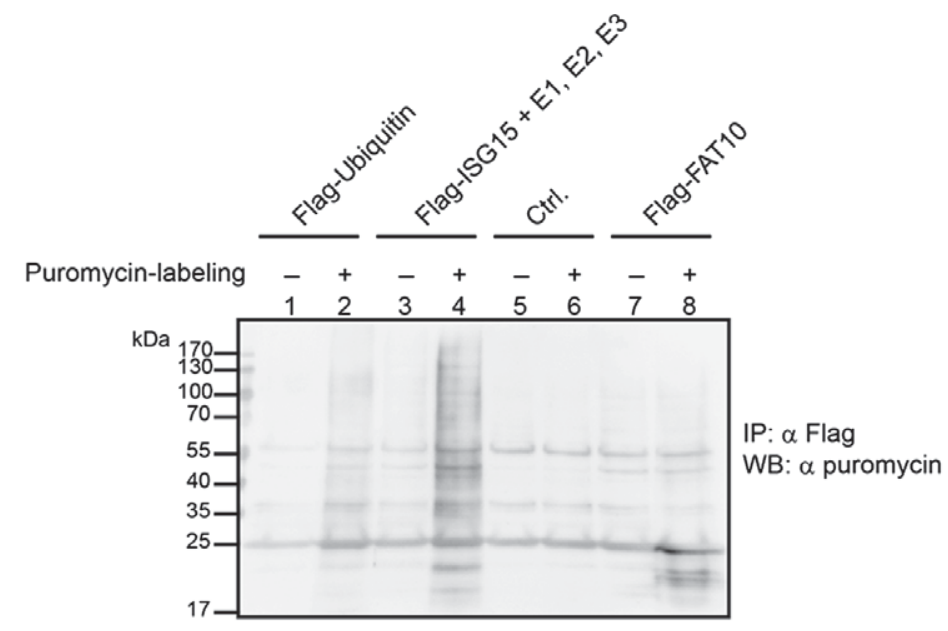

B
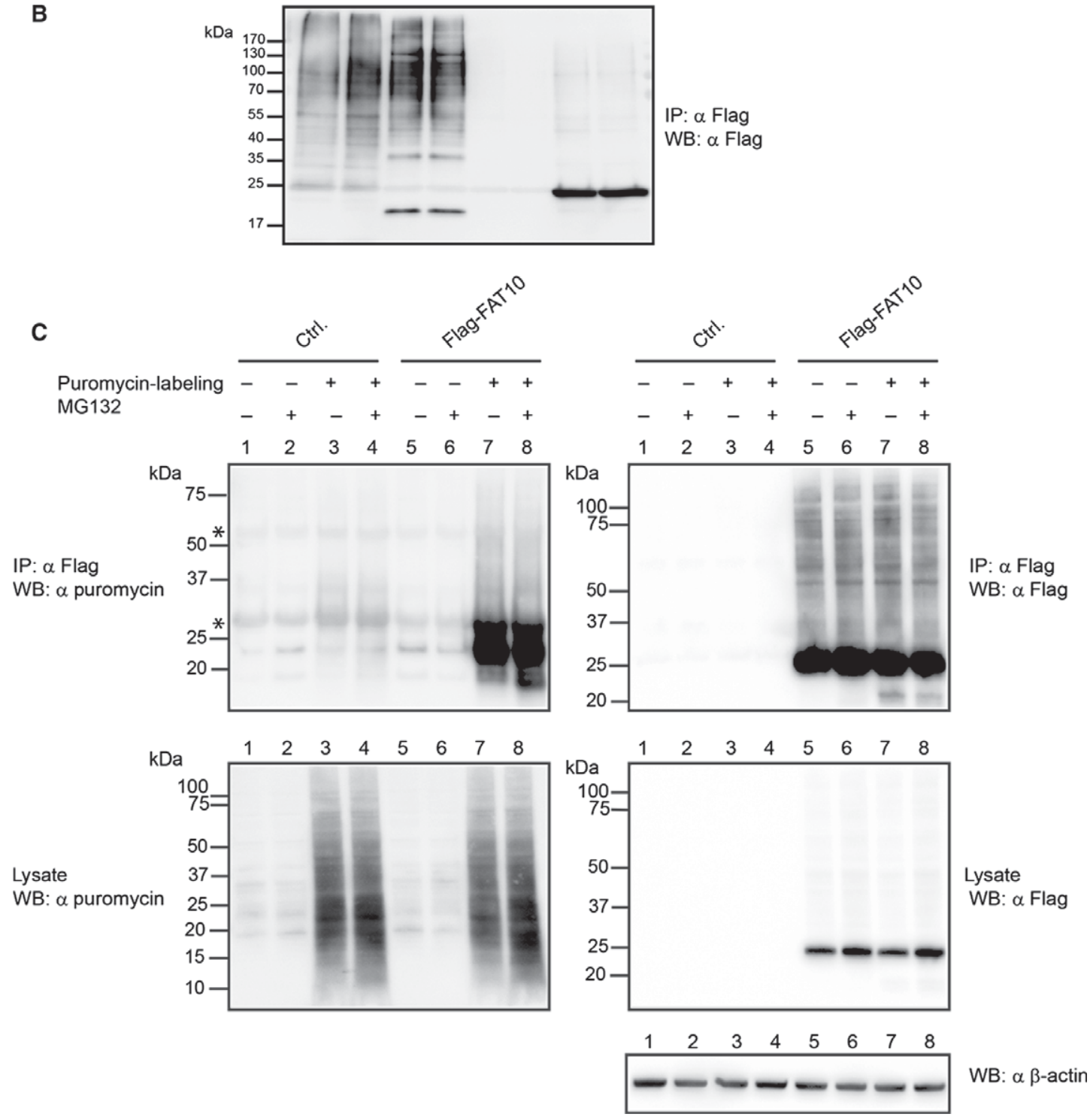
puromycin-positive conjugates were detectable under the same experimental conditions for FAT10 (Fig. 2A/ B lanes 7 and 8). Merely monomeric FAT10 was puromycin-labeled (bottom of lane 8), but such truncated FAT10 polypeptides cannot be conjugated to substrate proteins because this requires the $\mathrm{C}$-terminal glycine residue of FAT10. However, when we used three times more cells per immunoprecipitation, FAT10ylation of puromycin-labeled polypeptides was reproducibly detectable (Fig. 2C, lanes 7 and 8) when compared to unlabeled cells (Fig. 2C, lanes 5 and 6) or untransfected control cells (Fig. 2C, lanes 1-4). We tested whether the treatment of the cells with the proteasome inhibitor, MG132, $1 \mathrm{~h}$ before the start of the 10-min puromycin-labeling period would lead to an accumulation of Flag-FAT10 conjugates. This was detectable in the total lysates of Flag-FAT10-transfected cells (Fig. 2C, lane 8) suggesting that these puromycinlabeled polypeptides were rapidly degraded by the proteasome. Taken together, the data shown in Fig. $2 \mathrm{C}$ corroborate the notion that FAT10 is conjugated to newly translated polypeptides.

\section{Ubiquitin, ISG15, and FAT10 associate with purified ribosomes}

Finally, we were interested to investigate whether FAT10 associates with ribosome-associated nascent polypeptides as it has been shown for ubiquitin [6]. In the case of ISG15, Huibregtse and colleagues showed the ISG15-specific E3-ligase Herc5 fractionated with ribosomes in sucrose gradients [7].

To test the association of FAT10 or ISG15 conjugates with purified ribosomes, we transfected HEK293T cells either with expression constructs for Flag-ISG15 combined with its E1, E2, and E3 enzymes (Fig. 3A), or for HA-Ub (Fig. 3B), or for Flag-FAT10 (Fig. 3C), and then purified ribosomes from the postmitochondrial supernatant (denoted as input). The percentage of input in relation to the amount of purified ribosomes is indicated in each panel (Fig. 3A-C). Purification of ribosomes was verified by the presence of the $60 \mathrm{~S}$ ribosomal subunit Rpl7 and the lack of GAPDH signals in purified ribosome samples compared to total cytosol samples (input).

As shown in Fig. 3A, Flag-ISG15 conjugates were detectable in ribosome samples. The E3 ligase for human ISG15, Herc5, could also be detected in ribosome samples as previously reported [7], and was even enriched compared to the cytosolic input. Furthermore, HA-Ub conjugates coprecipitated with ribosomes (Fig. 3B) as previously described [6] but to a lesser extent than ISG15. In addition, we detected minimal amounts of the ubiquitin-activating E1 enzyme (Ube1) associated with ribosomes. The ribosome-associated E3 ligase listerin (Ltn)1, however, which was reported to be involved in cotranslational nascent chain degradation, was enriched in the ribosome preparations.

We also observed Flag-FAT10 and its conjugates to be associated with ribosomes, similar to the other two modifiers (Fig. 3C). The appearance of the smear of FAT10 conjugates in total lysates (Fig. 3C, lane 4) and ribosome preparation (Fig. 3C, lane 2) is quite similar arguing against the possibility that the FAT10 conjugates in the ribosome preparation are merely composed of FAT10ylated ribosome subunits. The conjugation enzymes for FAT10 known to date, that is, the activating enzyme Uba6 and the conjugating enzyme Use1, were detectable in the total lysate but not in the ribosome preparation suggesting that they are not bound to $80 \mathrm{~S}$ ribosomes. As no E3 ligase has been described for FAT10 conjugation to date, we could not test ribosome association of such a putative enzyme in analogy to Herc5.

\section{Discussion}

The fundamental role of the UPS in MHC-I peptide presentation has been widely accepted although a contribution of RDPs as a primary source for peptide supply is still debated [1,5]. Irrespective of the actual percentage of translation products that can be considered retirees or RDPs, there is a consensus that both species feed into the antigen processing pathway.

Antigen processing was initially suggested to be a pathway in which FAT10 might be functionally involved [9]. Supporting evidence was provided first of all because FAT10 is encoded in the MHC locus and cytokine inducible. Additionally, FAT10 expression is strongly induced during DC and medullary thymic epithelial cell maturation $[17,18]$ when antigen processing is upregulated in these cells $[19,20]$. Indeed, two studies revealed a role of FAT10 in degradation and presentation of model substrates for MHC class I presentation $[10,11]$. Previously, FAT10 was shown to target proteins for degradation in a ubiquitin-independent manner [18]. Furthermore, both modifiers showed differences in their way of proteasomal targeting, for example, the process of delivery and docking to the $19 \mathrm{~S}$ regulator [21]. These observations led to the hypothesis that FAT10 might fulfill a role in antigen processing.

The observation that FAT10 associates with purified ribosomes (Fig. 3C) implicates that the observed modification of newly translated proteins (Fig. 1D) might be carried out cotranslationally. However, a critical point for both experiments might be the upcoming 

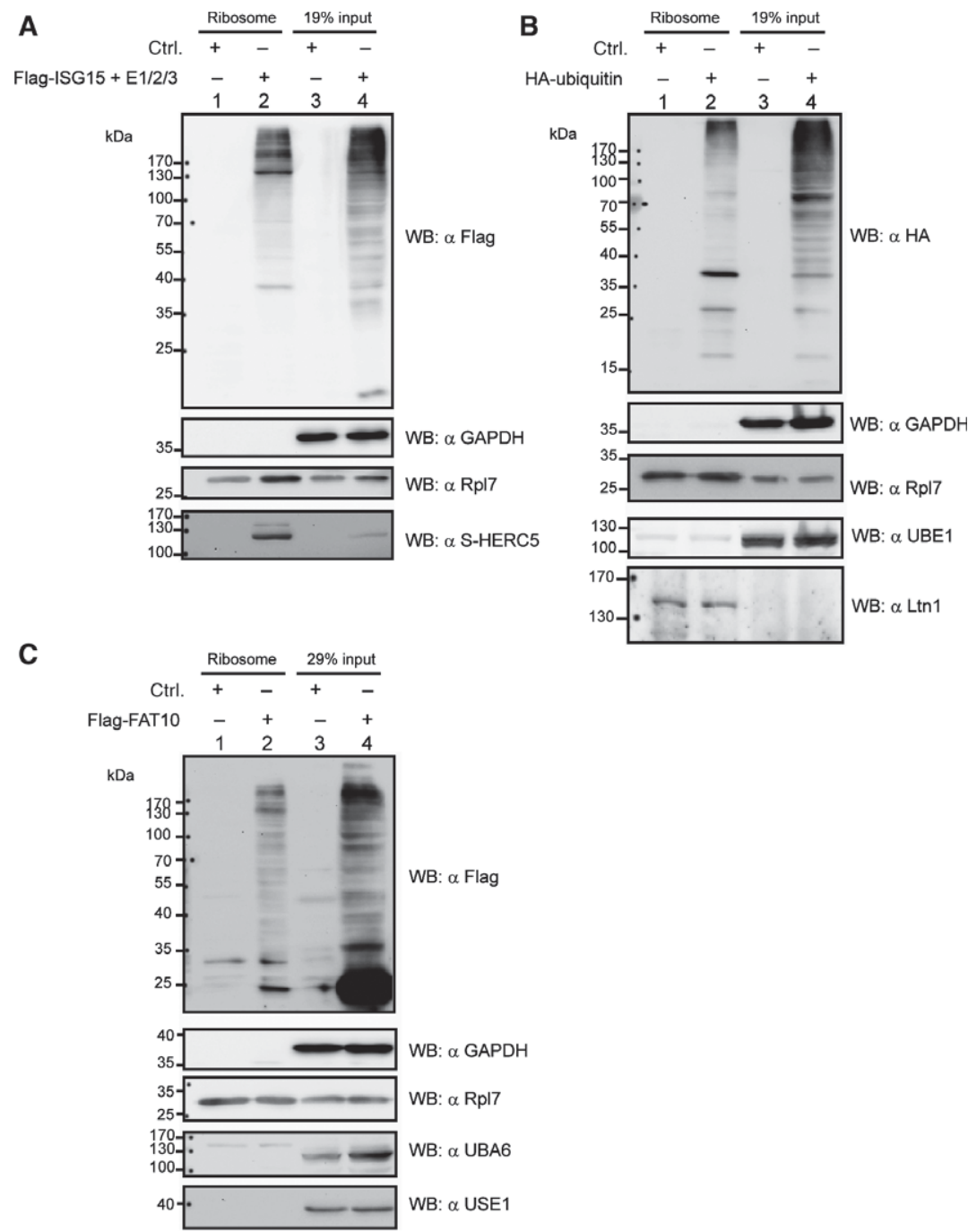

Fig. 3. ISG15, ubiquitin, and FAT10 are associated with purified ribosomes. HEK293T cells were transiently transfected with expression plasmids for either (A) Flag-ISG15-GG together with its corresponding E1, E2, E3 enzymes, Ube1L, UbcH8, and S-Herc5, or (B) with HAubiquitin-GG or (C) with HIS-Flag-FAT10-GG as indicated above each panel. After $24 \mathrm{~h}$ of transient expression, cells were lysed, centrifuged, and load samples (input) were taken from the postmitochondrial fraction. Ribosomes were purified from this fraction via a sucrose cushion (ribosome). Samples were analyzed by immunoblot with antibodies specific for Flag, HA, Herc5, UBE1, listerin (Ltn1), UBA6, and USE1 as indicated. Detection of GAPDH and the ribosome subunits Rpl7 served as controls.

awareness that apparently a certain percentage of translation takes place in the nucleus [22]. This observation even led to the hypothesis that this might be a relevant niche for antigenic peptide generation [23]. If so, results obtained in Figs 1 and 3 neglect this fraction of newly synthesized proteins because our experiments were carried out without ionic detergents. This applies to large aggregates as well, which also may contain newly translated proteins as previously reported [3]. Larger protein aggregates were probably not fully dissolved under the lysis conditions used in our study.
To verify the covalent conjugation to newly translated proteins (Fig. 1), we performed puromycin-labeling experiments (Fig. 2). We expected the strongest puromycin staining for ISG15 conjugates and this is indeed what we observed in Fig. 2A. Ubiquitin became conjugated to puromycin-labeled polypeptides to a lesser extent and FAT10ylation of puromycylated polypeptides became only visible when threefold more cells were used per immunoprecipitation (Fig. 2C). The fact that only low puromycin signals were detectable for FAT10 is probably due to a low sensitivity of the detection system and because the low conjugates to 
monomer ratio for FAT10 as compared to ubiquitin and ISG15 (Fig. 2B).

To find FAT10 to be conjugated to newly translated polypeptides (Fig. 1D) and to be associated with ribosomes (Fig. 3C) is consistent with a potential role of this modifier in proteasomal processing of newly translated proteins. The open question if an E3 ligase mediates FAT10 modification remains a high priority topic. Are there multiple ligases preserving specificity for different substrates or is FAT10 maybe promiscuously conjugated to many different substrates by a single E3 ligase, as previously reported for ISG15? In the case of ISG15, Huibregtse and coworkers provided an attractive explanation which states that ISGylation of newly synthesized virus proteins interferes with viral particle formation [7]. The preference of ISGylation for any newly translated protein at the ribosome might explain why one E3 ligase (HERC5) can modify such a vast number of ISGylation substrates. As FAT10 is not preferentially linked to newly translated proteins, such a scenario may not apply to FAT10. In the case of ubiquitin, there are several E3 ligases known to mediate ubiquitylation of RDPs. Interestingly, many of them have been shown to be associated with specific quality control pathways. The E3 ubiquitin ligase CHIP, for example, targets misfolded chaperone substrates toward proteasomal degradation [19]. Other E3 ligases like Ltn1, Hel2, Upf1, Ubr1, and Not4 have been proposed to play a role in degradation of nascent chains during different cotranslational quality control pathways [2]. This raises the question whether also FAT10 conjugation can be assigned to a specific subpopulation of newly translated proteins. Importantly, there is no evidence that FAT10 might target nascent chains of stalled ribosomes, as a previous MS-analysis of endogenous FAT10 substrates did not reveal an enrichment of proteins that migrated with unexpected small sizes in SDS/PAGE [20]. It seems therefore rather likely that newly translated FAT10 substrates represent DRiPs. Or they are no RDPs at all but a so far undefined group of proteins which rapidly acquire a stable conformation, as it has been shown for ISG15 [7]. At least the described function of FAT10 as a fast and direct targeting mechanism for proteasomal degradation implicates that FAT10 substrates at the ribosome may become rapidly degraded [18]. In addition, it is also thinkable that FAT10 is targeting these substrates to aggresome-like induced structures (ALIS). In DCs, where FAT10 is upregulated during maturation [17], these structures are called dendritic ALIS (DALIS) and have been suggested to function as antigen storage compartments
[24]. Interestingly, FAT10 has previously been shown to interact with two important mediators of aggregate formation, HDAC6 and p62 [20,25]. Moreover, FAT10 colocalizes with the autophagy protein LC3B [26]. However, in spite of these considerations, direct evidence for a function of FAT10 in cotranslational quality control is still lacking.

In summary, this study introduces FAT10 as a third modifier that is conjugated to newly translated proteins, besides ubiquitin and ISG15 [6,7], thereby suggesting unexpected parallels between these three conjugation machineries.

\section{Acknowledgements}

We thank Peter Walter for the contribution of puromycin-specific polyclonal antibody. We also thank Jon M Huibregtse for pcDNA-Ube1L [14], pcDNAUbcH8 [14], pc3xFLAG-ISG15-C0 [7] plasmids, Klaus-Peter Knobeloch for the contribution of pTriEx-2-S-hHerc5 [15], as well as Michael Basler for the constructs pcDNA3.1-HA-ubiquitinK48R $\Delta \mathrm{GG}$ (Ub-GA) and pcDNA3.1-HA-ubiquitin (Ub-GG) [8]. This study was supported by the German Research Foundation (DFG) Collaborative Research Center SFB969, project C01 and the Velux Foundation (grant 1029). VS received a stipend from the Graduate School Chemical Biology at the University of Konstanz. AB was a member of the DFG Research Training Group 1331 at the University of Konstanz.

\section{Author contributions}

VS designed, performed, and interpreted the experiments and wrote the manuscript; $\mathrm{AB}$ performed ribosome purifications; VS, AB, and AA performed puromycin-labeling experiments; and $\mathrm{MG}$ conceived and supervised the project, acquired funding, designed and interpreted the experiments, and refined the manuscript.

\section{References}

1 Anton LC and Yewdell JW (2014) Translating DRiPs: MHC class I immunosurveillance of pathogens and tumors. J Leukoc Biol 95, 551-562.

2 Wang F, Canadeo LA and Huibregtse JM (2015) Ubiquitination of newly synthesized proteins at the ribosome. Biochimie 114, 127-133.

3 Schubert U, Anton LC, Gibbs J, Norbury CC, Yewdell JW and Bennink JR (2000) Rapid degradation of a large fraction of newly synthesized proteins by proteasomes. Nature 404, 770-774. 
4 Vabulas RM and Hartl FU (2005) Protein synthesis upon acute nutrient restriction relies on proteasome function. Science 310, 1960-1963.

5 Rock KL, Farfan-Arribas DJ, Colbert JD and Goldberg AL (2014) Re-examining class-I presentation and the DRiP hypothesis. Trends Immunol 35, 144-152.

6 Wang F, Durfee LA and Huibregtse JM (2013) A cotranslational ubiquitination pathway for quality control of misfolded proteins. Mol Cell 50, 368-378.

7 Durfee LA, Lyon N, Seo K and Huibregtse JM (2010) The ISG15 conjugation system broadly targets newly synthesized proteins: implications for the antiviral function of ISG15. Mol Cell 38, 722-732.

8 Hipp MS, Kalveram B, Raasi S, Groettrup M and Schmidtke G (2005) FAT10, a ubiquitin-independent signal for proteasomal degradation. Mol Cell Biol 25, 3483-3491.

9 Basler M, Buerger S and Groettrup M (2015) The ubiquitin-like modifier FAT10 in antigen processing and antimicrobial defense. Mol Immunol 68, 129-132.

10 Schliehe C, Bitzer A, Van Den Broek M and Groettrup M (2012) Stable antigen is most effective for eliciting CD8+ T-cell responses after DNA vaccination and infection with recombinant vaccinia virus in vivo. J Virol 86, 9782-9793.

11 Ebstein F, Lehmann A and Kloetzel PM (2012) The FAT10- and ubiquitin-dependent degradation machineries exhibit common and distinct requirements for MHC class I antigen presentation. Cell Mol Life Sci 69, 2443-2454.

12 Chiu Y, Sun Q and Chen Z (2007) E1-L2 activates both ubiquitin and FAT10. Mol Cell 27, 1014-1023.

13 Aichem A, Pelzer C, Lukasiak S, Kalveram B, Sheppard PW, Rani N, Schmidtke G and Groettrup M (2010) USE1 is a bispecific conjugating enzyme for ubiquitin and FAT10, which FAT10ylates itself in cis. Nat Commun 1, 13.

14 Zhao C, Denison C, Huibregtse JM, Gygi S and Krug RM (2005) Human ISG15 conjugation targets both IFN-induced and constitutively expressed proteins functioning in diverse cellular pathways. Proc Natl Acad Sci U S A 102, 10200-10205.

15 Ketscher L, Basters A, Prinz M and Knobeloch KP (2012) mHERC6 is the essential ISG15 E3 ligase in the murine system. Biochem Biophys Res Commun 417, 135-140.
16 Marchese A and Benovic JL (2001) Agonist-promoted ubiquitination of the $\mathrm{G}$ protein-coupled receptor CXCR4 mediates lysosomal sorting. J Biol Chem 276, 45509-45512.

17 Lukasiak S, Schiller C, Oehlschlaeger P, Schmidtke G, Krause P, Legler DF, Autschbach F, Schirmacher P, Breuhahn K and Groettrup M (2008) Proinflammatory cytokines cause FAT10 upregulation in cancers of liver and colon. Oncogene 27, 6068-6074.

18 Schmidtke G, Aichem A and Groettrup M (2014) FAT10ylation as a signal for proteasomal degradation. Biochim Biophys Acta 1843, 97-102.

19 Connell P, Ballinger CA, Jiang J, Wu Y, Thompson LJ, Hohfeld J and Patterson C (2001) The co-chaperone CHIP regulates protein triage decisions mediated by heat-shock proteins. Nat Cell Biol 3, 93-96.

20 Aichem A, Kalveram B, Spinnenhirn V, Kluge K, Catone N, Johansen T and Groettrup M (2012) The proteomic analysis of endogenous FAT10 substrates identifies p62/SQSTM1 as a substrate of FAT10ylation. $J$ Cell Sci 125, 4576-4585.

21 Rani N, Aichem A, Schmidtke G, Kreft SG and Groettrup M (2012) FAT10 and NUB1L bind to the VWA domain of Rpn10 and Rpn1 to enable proteasome-mediated proteolysis. Nat Commun 3, 749.

22 David A, Dolan BP, Hickman HD, Knowlton JJ, Clavarino G, Pierre P, Bennink JR and Yewdell JW (2012) Nuclear translation visualized by ribosomebound nascent chain puromycylation. J Cell Biol 197, 45-57.

23 Yewdell JW and David A (2013) Nuclear translation for immunosurveillance. Proc Natl Acad Sci U S A 110, 17612-17613.

24 Pierre P (2005) Dendritic cells, DRiPs, and DALIS in the control of antigen processing. Immunol Rev 207, 184-190.

25 Kalveram B, Schmidtke G and Groettrup M (2008) The ubiquitin-like modifier FAT10 interacts with HDAC6 and localizes to aggresomes under proteasome inhibition. J Cell Sci 121, 4079-4088.

26 Spinnenhirn V, Farhan H, Basler M, Aichem A, Canaan A and Groettrup M (2014) The ubiquitin-like modifier FAT10 decorates autophagy-targeted Salmonella and contributes to Salmonella resistance in mice. J Cell Sci 127, 4883-4893. 